\title{
The Superior Outcome of Collagen Nanosilver in Increasing Macrophage and Collagen on Deep Dermal Burn Wound Healing
}

\author{
Anggun Ari Mukti ${ }^{1}{ }^{(\mathbb{D}}$, Awal Prasetyo ${ }^{2} \mathbb{D}$, Yan Wisnu Prajoko ${ }^{2} \mathbb{D}$, Najatullah Najatullah $^{2} \mathbb{D}$, Neni Susilaningsih $^{3} \mathbb{D}$ \\ ${ }^{1}$ Department of General Surgery, Faculty of Medicine, Diponegoro University, Semarang, Indonesia; ${ }^{2}$ Department of Biomedical \\ Science, Faculty of Medicine, Diponegoro University, Semarang, Indonesia; ${ }^{3}$ Department of Plastic Surgery, Faculty of Medicine, \\ Diponegoro University, Semarang, Indonesia
}

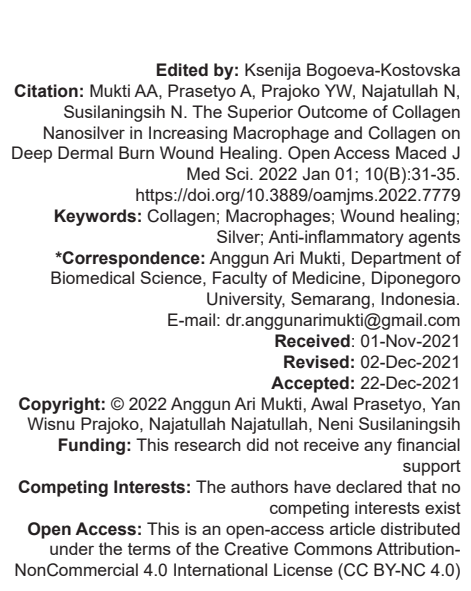

\begin{abstract}
AIM: This study aims to prove the superior outcome of collagen nanosilver in increasing macrophage and collagen on deep dermal burn wound healing.

METHODS: This study was a randomized control trial design. All animals used were male Sprague Dawley rats aged 12-14 weeks old and weighed about $200-250 \mathrm{~g}$. Each rat gets four site injuries and is given treatments. The treatment group includes: I (negative control using $\mathrm{NaCl} 0.9 \%$ ), II (positive control using silver sulfadiazine cream), III (treatment with nanosilver collagen cream 1\%), and IV (treatment with collagen nanosilver cream $2 \%$ ). Macrophages counts were assessed using IHC F40/80 and collagen density was assessed using Masson's trichrome staining.

RESULTS: Macrophage on day 6 showed a significant difference as seen in group III and group IV. A significan difference was also obtained between macrophage on day 3 and day 6 from group IV, likewise between collagen between day 3 and day 6 from group III and IV. Collagen nanosilver acts as an antimicrobial and anti-inflammatory agent by increasing the mechanism of activation and migration of macrophages to the site of injury. Moreover, this formula modulated the collagen deposition, which forms the tissue fibers which ultimately provide a conducive
\end{abstract} environment for wound healing.

CONCLUSION: Collagen nanosilver has a superior outcome in the increasing macrophage cell count and collagen density of deep dermal burn wound healing.

\section{Introduction}

Burn injury is a form of destructive trauma, which often results in significant morbidity and mortality rates, causes an emotional disturbance, and demoted quality of life [1], [2]. According to the data from the World Health Organization [3], over 300.000 patients worldwide die each year from fire-related burn injury. Burn trauma has a contribution of $0.7 \%$ or almost 18 million population in the world. In 2015, the Ministry of Health in Indonesia recorded that burn trauma ranked $6^{\text {th }}$ for accidental injuries [4]. The high medical costs of acute burn injury followed by the high costs of rehabilitation and loss of income [1].

The main principles for the management of deep dermal burn injury are assessment of the burn depth, wound care, and prevention of infection, provide a conducive environment to facilitate the process of tissues growth [5]. Silver sulfadiazine is the gold standard in topical burn treatment, since the findings of these adverse effects, involving the inability to penetrate the eschar, leucopenia, and toxicity effect on infant and pregnant women [6]. The recent findings develop to accelerate the wound healing processes. Recently, the use of silver nanoparticles gained interest as an antiseptic and anti-inflammatory agent in dressings [7]. Collagen applications have been widely used as an effective and safe debridement agent in reducing wound healing time and reducing pain without increasing the risk of infection [8]. The formulations of nanoparticle silver and collagen were fabricated to provide an optimal wound healing environment of deep dermal burn injury using the advantages of nanosilver particle and collagen dressings. The nanosilver technology has been recommended for second degree burn wound [9].

To address the issue, this study aims to prove the effectiveness of the collagen nanosilver cream compared to silver sulfadiazine as standard therapy in a topical treatment to accelerate the healing of deep dermal burn injury on Sprague Dawley rats through the biomedical parameters of macrophage and collagen. 


\section{Methods}

\section{Experimental burn wound}

All animals used were male Sprague Dawley rats aged 12-14 weeks old and weighed about 200-250 g. Sprague Dawley rats were obtained from Animal House, Faculty of Medicine, Universitas Diponegoro (Semarang, Central Java, Indonesia). All procedures were reviewed and conducted in accordance with the Declaration of Helsinki 1975. This study has received approval from Ethics Committee of the Faculty of Medicine, Universitas Diponegoro (No. 121/KE/UNDIP/2021).

The rats were divided into four groups (Table 1); group I (negative control), group II (positive control), group III (treatment with 1\% collagen nanosilver cream), and group IV (treatment treated with $2 \%$ collagen nanosilver cream). The induction of deep dermal burn injury was carried out by prior anesthesia in rats using a combination intraperitoneal injection of ketamine (50 $\mathrm{mg} / \mathrm{kg}$ ) (Dexamedica, Palembang, Indonesia) and xylazine $(5 \mathrm{mg} / \mathrm{kg}$ ) (Dexamedica, Palembang, Indonesia). The rats were induced deep dermal burn injury using a notch heater with $1 \mathrm{~cm}$ diameter and $150^{\circ}$ temperature for $20 \mathrm{~s}$. The variables examined in this study included the macrophage cell count and collagen density. The results of the study were assessed on day 3 and day 6 of the treatment.

\section{Table 1: Experimental group treatment}

\begin{tabular}{ll}
\hline Group & Treatment \\
\hline Group I & The rats were burn wound induced and given $0.9 \% \mathrm{NaCl}$ \\
(negative control) & topical \\
Group II & The rats were burn wound induced and treated with topical \\
(positive control) & silver sulfadiazine cream \\
Group III & The rats were burn wound induced and treated with topical \\
& $\begin{array}{l}1 \% \text { collagen nanosilver cream } \\
\text { Group IV }\end{array}$ \\
& The rats were burn wound induced and treated with topical \\
& $2 \%$ collagen nanosilver cream \\
\hline
\end{tabular}

\section{Collagen nanosilver cream}

The manufactured of collagen nanosilver is carried out at the Integrated Laboratory of Universitas Diponegoro (Semarang, Indonesia). The concentration of collagen nanosilver is made into two stage doses, involved $1 \%$ and $2 \%$ concentration.

\section{Histopathological examination}

Skin tissue that was fixed with $4 \%$ paraformaldehyde buffer was dehydrated using graded alcohol and xylene, then paraffinized and cut to a 5 um thickness using a spinning microtome (Leica, Weitzar, Germany). The result of the cuts was then placed on a glass object and dyed with Masson's trichrome. Histopathological changes in skin tissue were interpreted under a microscope (Olympus, Tokyo, Japan).

\section{Immunohistochemistry examination}

After the rats were sacrificed by intraperitoneal injection of $10 \%$ chloral hydrate, then the skin was evacuated, fixed in $4 \%$ paraformaldehyde buffer for immunohistochemistry tests of skin tissue. The tissue that has been inserted into the next fixation fluid is dehydrated using graded alcohol and xylene, then paraffinized and cut to a thickness of 5 um using a rotary microtome (Leica, Illinois, USA). The following step, placed on a coated-object glass. Then, the tissues were rehydrated using xylene and alcohol with a gradient of $96 \%, 90 \%, 80 \%$, and $70 \%$ concentrations and rinsed with tap water. The next step was carried out with the retrieval antigen using the HIER (Heat Induced Epitope Retrieval) method, where the slides were put in a citrate buffer solution, then heated at $95^{\circ} \mathrm{C}$ for $60 \mathrm{~min}$. Then, IHC F4/80 1: 700 (Cloud Clone, Hangzhou, PRC) antibody was stained, followed by overnight incubation at $4^{\circ} \mathrm{C}$. The next step was staining with a secondary antibody, Biotinylated-HRP (Horseradish Peroxidase), incubation for $1 \mathrm{~h}$, at room temperature. Furthermore, chromogen was administered. Next, the dehydration process was again carried out using a concentration of alcohol and xylene.

\section{Statistical analysis}

All data were presented as mean \pm standard deviation, and statistical analysis was performed with the SPSS 25 (IBM, Armonk, USA) program. One-way ANOVA accompanied by a post hoc analysis was carried out to assess the difference in mean expression levels of collagen density and macrophage count. $p<0.05$ was determined as an indication that there was a significant difference in mean levels.

\section{Results}

The tissue samples were examined by a histologist. Macrophage cells counted per 100 inflammatory cells in the inflamed area through a microscope, then confirmed by immunohistochemical examination using IHC F4/80. Collagen density counted per 5 visual areas through a microscope.

Microscopic imaging examination shows macrophages cell count and collagen density between the 4 groups during treatment days 3 and 6 . The highest macrophage cell count was obtained in group IV then followed by group III. The microscopic imaging of collagen between the 4 groups during treatment days 3 and 6 showed that the highest collagen density was yield in group IV.

Figure 1 showed the characteristic of macrophage cell count on day 3 and day 6 . The highest 


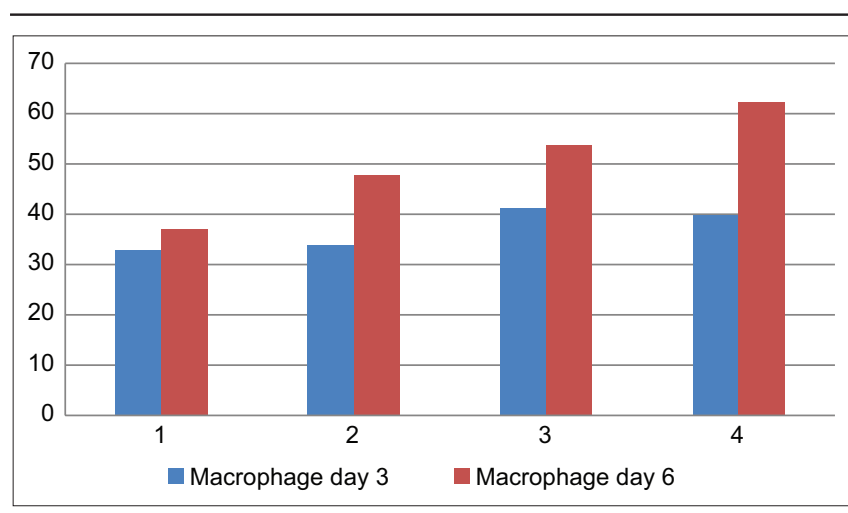

Figure 1: Characteristic of macrophage on day 3 and day

macrophage on day 3 was obtained in group III and group IV. The highest macrophage on day 6 was seen in group IV and group III. Figure 2 showed the characteristic of collagen density on day 3 and day 6 . The highest collagen on day 3 was obtained in group IV. The highest collagen on day 6 was seen in group IV.

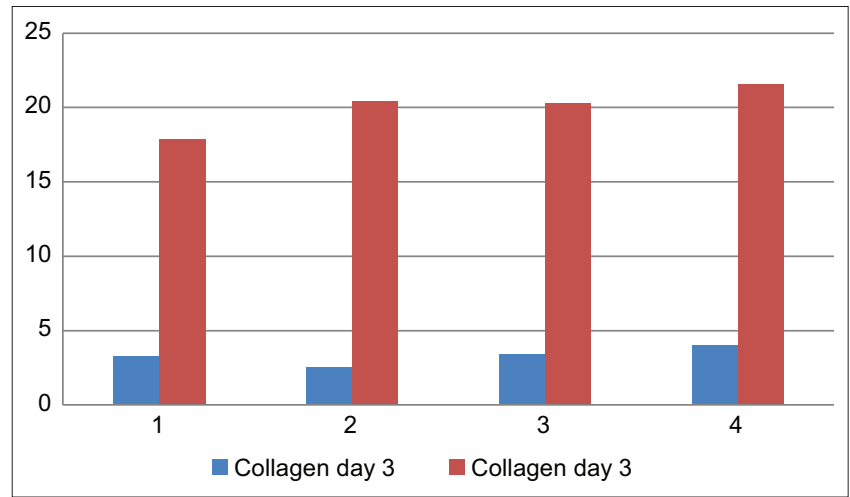

Figure 2: Characteristic of collagen on day 3 and day 6

Table 2 showed the significant result obtained in macrophage on day 6 from group III-I $(p=0,004)$, IV-I $(p=0,000)$, and IV-II $(p=0,013)$. From posthoc Games Howell test in Table 3, a significant difference was obtained between macrophages on day 3 and day 6 from group IV $(p=0,001)$. Significant difference also seen on between collagen on day 3 and day 6 from group III $(p=0,000)$ and IV $(p=0,000)$.

Table 2: The difference of collagen and macrophage between treatment group

\begin{tabular}{llllll}
\hline Variable & Group & D3 group I & D3 group II & D3 group III & D3 group IV \\
\hline Collagen & D3 group I & - & 1.000 & 1.000 & 1.000 \\
& D3 group II & 1.000 & - & 1.000 & 0.174 \\
& D3 group III & 1.000 & 1.000 & - & 1.000 \\
& D3 group IV & 1.000 & 0.174 & 1.000 & - \\
Macrophage & D3 group I & - & 1.000 & 0.820 & 1.000 \\
& D3 group II & 1.000 & - & 1.000 & 1.000 \\
& D3 group III & 0.820 & 1.000 & - & 1.000 \\
& D3 group IV & 1.000 & 1.000 & 1.000 & - \\
\hline Variable & Group & D6 group I & D6 group II & D6 group III & D6 group IV \\
\hline Collagen & D6 group I & - & 1.000 & 1.000 & 0.553 \\
& D6 group II & 1.000 & - & 1.000 & 1.000 \\
& D6 group III & 1.000 & 1.000 & - & 1.000 \\
Macrophage & D6 group IV & 0.553 & 1.000 & 1.000 & - \\
& D6 group I & - & 0.089 & $0.004^{*}$ & $0.000^{*}$ \\
& D6 group II & 0.089 & - & 0.894 & $0.013^{*}$ \\
& D6 group III & $0.004^{*}$ & 0.894 & - & 0.298 \\
& D6 group IV & $0.000^{*}$ & $0.013^{*}$ & 0.298 & - \\
\hline D3: Third day of treatment; D6: Sixth day of treatment; *:post hoc Bonferroni test, p<0.05.
\end{tabular}

\section{Discussion}

This research proves that the collagen nanosilver scaffold is better than silver sulfadiazine in increasing macrophage cell count on deep dermal burn wound healing. Wound healing occurs in three phases, involving the inflammatory phase, the proliferation phase, and the remodeling phase. Macrophages are the main immune cells with their important roles in phagocytes and clearing the site of injury from necrotic tissue, pathogenic microorganism,

Table 3: The difference of collagen and macrophage between treatment day

\begin{tabular}{llllll}
\hline Variable & Group & D6 group I & D6 group II & D6 group III & D6 group IV \\
\hline Collagen & D3 group I & $0.007^{* *}$ & $0.005^{* *}$ & $0.000^{* *}$ & $0.000^{* *}$ \\
& D3 group II & $0.008^{* *}$ & $0.005^{* *}$ & $0.000^{* *}$ & $0.001^{* *}$ \\
& D3 group III & $0.009^{* *}$ & $0.006^{* *}$ & $0.000^{* *}$ & $0.001^{* *}$ \\
& D3 group IV & $0.007^{* *}$ & $0.004^{* *}$ & $0.000^{* *}$ & $0.000^{* *}$ \\
Macrophage & D3 group I & 1.000 & 0.090 & $0.003^{*}$ & $0.000^{*}$ \\
& D3 group II & 1.000 & 0.156 & $0.005^{*}$ & $0.000^{*}$ \\
& D3 group III & 1.000 & 1.000 & 0.327 & $0.003^{*}$ \\
& D3 group IV & 1.000 & 1.000 & 0.156 & $0.001^{*}$ \\
\hline \multicolumn{7}{l}{ D3: Third day of treatment; D6: Sixth day of treatment; *:Post-hoc Bonferroni test, p<0.05; **: Post-hoc }
\end{tabular}
Games Howell, $p<0.05$.

and extracellular debris. Monocytes as a subset of white blood cells, which are rapidly recruited from the circulatory system to the site of injury. Monocytes may transiently persist as monocytes secreting proinflammatory and angiogenic factors, or differentiate into macrophages to support tissue typical macrophages at the wound site to begin extracellular debridement [10], [11]. Macrophages migrate into the wound site of injury within 48-96 h after injury to help neutrophils complete debridement in order to terminate the inflammatory response [12]. The action mechanism of nanosilver collagen is more reactive than the bulk silver form caused by their nanometer-sized particles. The high surface to volume ratio of the nanoparticles is another advantage as it allows the particles to remain effective even at a very low concentration [13], [14].

Macrophages secrete various cytokines and chemokines to stimulate cell proliferation and collagen deposition, promoting vascularization and granulation. There are two various activation mechanisms of macrophages, including classical macrophage activation (M1) as immune and inflammatory cells in the earlier phase of wound healing, and alternative macrophage activation (M2) as the major repairing cells and dominate at the later stage of wound healing. Nanoparticle silver as an anti-bacterial and anti-inflammatory agent regulates the macrophage activation on-site injury and promoted the inflammation phase to accelerate wound healing [10]. Collagen provides a template for the infiltration of fibroblasts, macrophages, and lymphocytes and attracts additional monocytes to the wound, thus increasing the debris removed and increasing neovascularization [15].

The research data also establish that the collagen nanosilver scaffold is better than silver sulfadiazine in increasing collagen density on deep 
dermal burn wound healing. Nanoparticle silver enhanced the differentiation of fibroblasts into myofibroblasts, resulting in faster wound contraction during the healing process. Collagen density in the treatment group using collagen nanosilver cream resulting the highest pattern compared to the control group. This finding conceived that this scaffold increasing the tensile on skin tissues and assembled the alignment of fibril which generated the wound healing tissue similar to normal skin. The collagen pattern according to a previous study which found collagen fibers formed by nanosilver are spaghetti and lentil, where the fiber deposition is neatly arranged. The characteristics of collagen fibers in the nanosilver collagen treatment group are equal to normal skin. This was concrete evidence that collagen nanosilver modulate collagen deposition in wound healing, and this also explained why the treatment group demonstrated prior wound healing rather than forming scars [16].

Collagen is a biomaterial that promotes wound healing through the deposition and organization of freshly formed fibers and granulation tissue in the wound bed, thus creating a conducive environment for wound healing. Collagen encourages fibroblast migration which subsequently produces reparative connective tissue. The final phase of the healing process involves the production, maturation, and degradation of collagen, the fibrils of which bridge the space between the edges of the injured tissue to form a scar of sizeable tensile and breaking strength. The force and functional adequacy of a scar depend on the native intensity and timing of collagen metabolism [15], [17].

The prior research support this study, which incorporates nanoparticle silver into collagen scaffold will preserve the mechanical properties and biocompatibility in skin fibroblast and keratinocyte structure. This action will promote skin regeneration [18].

\section{Conclusion}

Collagen nanosilver has a superior outcome in increasing macrophage cell count and collagen density of deep dermal burn wound healing on Sprague Dawley. Collagen nanosilver plays an important role in regulating macrophage activation, helps migrate macrophages, and improves the mechanism of phagocytosis in healing deep dermal burn wounds healing. This scaffold contributes in promoting the differentiation of fibroblasts to myofibroblasts, resulting in the acceleration of wound contractions. This material will encourage the wound healing progress through the deposition process, tissue fiber formation, and granulation tissue at the wound base, thereby producing a conducive environment to wound healing

\section{Acknowledgement}

The authors express their sincere gratitude to nanotechnology team of Diponegoro University for manufacturing the collagen nanosilver cream in this study.

\section{References}

1. Jeschke MG, van Baar ME, Choudry MA, Chung KK, Gibran NS Logsetty S. Burn Injury. Nat Rev Dis Primers. 2020;6(1):11. https://dx.doi.org/10.1038/s41572-020-0145-5

PMid:32054846

2. Kaddoura I, Abu-Sittah G, Ibrahim A, Karamanoukian R, Papazian N. Burn injury: Review of pathophysiology and therapeutic modalities in major burns. Ann Burns Fire Disasters. 2017;30(2):95-102. PMid:29021720

3. World Health Organization. A WHO Plan for Burn Prevention and Care. Geneva: World Health Organization; 2008. Available from: http://apps.who.int/iris/bitstream/handle/10665/97852/ 9789241596299_eng.pdf;jsessionid=36B45B30D51A107359C C1336 54158371?sequence=1 [Last accessed on 2021 Jan 24].

4. Indonesian Ministry of Health. Main Health Research 2018. Indonesian Health Research Foundation; 2018. p. 11-5.

5. Rowan MP, Cancio LC, Elster EA, Burmeister DM, Rose LF, Natesan S, et al. Burn wound healing and treatment: Review and advancements. Crit Care. 2015;19:243. https://dx.doi. org/10.1186/s13054-015-0961-2

PMid:26067660

6. Atiyeh BS, Costagliola M, Hayek SN, Dibo SA. Effect of silver on burn wound infection control and healing: Review of the literature. Burns. 2007;33(2):139-48. https://doi.org/10.1016/j. burns.2006.06.010

PMid:17137719

7. Wilkinson LJ, White R, Chipman JK. Silver and nanoparticles of silver in wound dressings: A review of efficacy and safety. J Wound Care. 2011;20(11):543-39. https://doi.org/10.12968/ jowc.2011.20.11.543 PMid:22240850

8. Pham $\mathrm{CH}$, Collier ZJ, Fang M, Howell A, Gillenwater TJ. The role of collagenase ointment in acute burns: A systematic review and meta-analysis. J Wound Care. 2019;28(Supppl 2):S9-15. https://dx.doi.org/10.12968/jowc.2019.28.Sup2.s9 PMid:30767636

9. Abadi AD, Vaheb M, Rakhshani MH, Tofighian T. Comparison of the effect of nanosilver spray and $1 \%$ silver sulfadiazine cream on the healing of second degree burn wound. Transl Biomed. 2018;9(1):141. https://dx.doi.org/10.21767/2172-0479.100141

10. You C, Li Q, Wang X, Wu P, Ho JK, Jin R, et al. Silver nanoparticle loaded collagen/chitosan scaffolds promote wound healing via regulating fibroblast migration and macrophage activation. Sci Rep. 2017;7(1):10489. https://doi.org/10.1038/ s41598-017-10481-0 PMid:28874692

11. Lateef Z, Stuart G, Jones N, Mercer A, Fleming S, Wise L. The cutaneous inflammatory response to thermal burn injury in a murine model. Int J Mol Sci. 2019;20(3):538. https://dx.doi. org/10.3390/ijms20030538 
PMid:30696002

12. Oka T, Ohta K, Kanazawa T, Nakamura KI. Interaction between macrophages and fibroblasts during wound healing of burn injuries in rats. Kurume Med J. 2016;62(3-4):59-66. https://doi. org/10.2739/kurumemedj.ms00003

PMid:27237937

13. Konop M, Damps T, Misicka A, Rudnicka L. Certain aspects of silver and silver nanoparticles in wound care: A minireview. J Nanomater. 2016;2016:761453. https://doi. org/10.1155/2016/7614753

14. Adhya A, Bain J, Ray O, Hazra A, Adhikari S, Dutta G, et al. Healing of burn wounds by topical treatment: A randomized controlled comparison between silver sulfadiazine and nanocrystalline silver. J Basic Clin Pharm. 2014;6(1):29-34. https:// dx.doi.org/10.4103/0976-0105.145776

PMid:25538469

15. Waghmare M, Shah H, Tiwari C, Makhija D, Desale J, Dwivedi $P$. Collagen dressings in the management of partial-thickness pediatric burns: Our experience. Indian J Burns. 2016;24(1):53-7. https://dx.doi.org/10.4103/0971-653X.195525

16. Kwan KH, Liu X, To MK, Yeung KW, Ho CM, Wong KK Modulation of collagen alignment by silver nanoparticles results in better mechanical properties in wound healing. Nanomedicine. 2011;7(4):497-504. https://doi.org/10.1016/j. nano.2011.01.003

PMid:21272666

17. Purna SK, Babu M. Collagen based dressings--a review. Burns. 2000;26(1):54-62. https://doi.org/10.1016/ s0305-4179(99)00103-5

PMid:10630321

18. Cardoso VS, Quelemes PV, Amorin A, Primo FL, Gobo GG, Tedesco AC, et al. Collagen-based silver nanoparticles for biological applications: Synthesis and characterization. J Nanobiotechnol. 2014;12(1):36. https://doi.org/10.1186/ s12951-014-0036-6

PMid:25223611 